\title{
Von kommunikativen Gattungen zu kommunikativen Formen: Konsequenzen des kommunikativen Konstruktivismus
}

\section{Einleitung}

Die Forschung zu kommunikativen Gattungen in der Linguistik ist untrennbar mit dem Namen Susanne Günthner verbunden. Der Begriff, der als ein Forschungsprogramm in der Soziologie entwickelt wurde, nahm mit ihr eine Relevanz auch für die Linguistik an. Daran waren natürlich auch andere Forschende beteiligt, nicht zuletzt Peter Auer, Elizabeth Couper-Kuhlen, Arnulf Deppermann, Helga Kotthoff oder Aldo di Luzio, doch war Susanne Günthner sicherlich die erste Linguistin, die sich empirisch mit dem Konzept beschäftigt hat. Der Grund liegt freilich darin, dass sie als Linguistin Teil des ersten DFG-finanzierten Gattungsprojektes war, das von Thomas Luckmann und Jörg Bergmann geleitet wurde. Dort durfte auch ich - während meiner Assistenzzeit in Sankt Gallen als wissenschaftliche Hilfskraft bei Angela Keppler, später dann als Assistent von Thomas Luckmann - mit ihr zusammenarbeiten. Die Zusammenarbeit nicht nur im gemeinsamen Schreiben, sondern auch in dialogischen Situationen (wie etwa Datensitzungen) mit Susanne bildete für mich wie ich mit einer persönlichen Note hinzufügen darf - das Musterbeispiel der interdisziplinären, kollegial-freundschaftlichen und geschlechtsübergreifenden Kooperation beim wissenschaftlichen Arbeiten. Dieser persönliche Hintergrund ist auch theoretisch folgenreich, denn wir beide führten nicht nur eine Reihe von empirischen Analysen kommunikativer Gattungen durch; zusammen verfassten wir mehrere konzeptionelle Artikel zur Theorie und Methodologie kommunikativer Gattungen (Günthner/Knoblauch 1994, 1995, 1996, 2000, 2007). Suchten wir in diesen Arbeiten zunächst zu bestimmen, wie die anfänglich eher programmatischen Überlegungen Luckmanns (1986) in unserer empirischen Forschung innerhalb der Projekte methodologisch umgesetzt wurden, so enthielten sie auch weitergehende theoretische Anregungen etwa zur Verbindung mit der interaktionalen Soziolinguistik und der Interaktionsforschung, wie sie etwa in der Ausweitung von zwei (Außen und Binnenstruktur) auf drei Analyseebenen (situative Realisierung) zum Ausdruck kam. 
Mit der zunehmenden Ausbreitung der „Gesprächslinguistik“ gewann diese mittlere Ebene des Konversationellen, Gesprächsartigen und Sequentiellen gerade in der Linguistik an Bedeutung. Sie erwies sich etwa in Günthners Analyse der Rolle des sozialen Geschlechts im Gespräch (Günthner/Kotthoff 1991; Günthner et al. 2012) wie auch in der Analyse der für die Linguistik so elementaren Grammatik (Günthner 2006) als überaus fruchtbar. Schon in unserem ersten gemeinsamen Aufsatz schlugen wir mit dem Konzept des kommunikativen Musters ein Konzept der Gattungsanalyse vor, das uns bis heute beschäftigt. So einleuchtend nämlich das Konzept der kommunikativen Gattungen als Verfestigung kommunikativen Handelns ist (wir werden es weiter unten erläutern), so sehr beschränkt sich der Begriff auf die sozusagen ausgeprägten Fixierungen: Dass Märchen, christliche Konversionserzählungen oder politische Reden verfestigt sind, scheint im Regelfall außer Frage zu stehen. ${ }^{1}$ Was aber, wenn sich die Verfestigung lediglich auf bestimme Aspekte kommunikativer Abläufe bezieht? Sind auch argumentative Gespräche (Knoblauch 1990), Belehrungssequenzen (Keppler/Luckmann 1991), Traumdarstellungen (Bergmann/Peräkylä i. d. B.) oder Vorwürfe (Günthner 2000) kommunikative Gattungen? Um darauf hinzuweisen, dass auch nur einzelne Aspekte - etwa prosodische Aspekte der Dissens- oder Wissensmarkierungen „verfestigt“ sein können, haben wir den Begriff des Musters vorgeschlagen.

Der Begriff des Musters eignet sich vorzüglich für Verfestigungen, die sich auf konversationelle, gesprächsartige Sequenzen beziehen, in denen Sprache in welchem Medium auch immer - Verwendung findet. Was aber ist mit jenen Abläufen, die nicht vorrangig, ja nicht einmal unbedingt sprachlich sind: So können lange Abläufe von Verkaufsinteraktionen, Dienstleistungen („Bedienen“) und Arbeits-, Sport- oder Familieninteraktionen (etwa mit Kleinkindern) ganz ohne Sprache auskommen, doch wäre es sicherlich falsch, sie nicht als „fixiert“ zu betrachten. ${ }^{2}$

So beiläufig die Frage erscheint, dreht es sich doch theoretisch um eine sehr grundlegende Entscheidung, die Luckmann sehr deutlich gemacht hat: Handelt es sich bei kommunikativen Gattungen um Verfestigungen, die sich nur auf „kommunikatives Handeln“ beziehen, das dem „Reden über“ entspricht und richtiges

1 Dieser Beitrag beruht sehr stark auf Überlegungen, die in einer umfänglichen Arbeit ausführlicher entfaltet wurden. Deswegen muss ich um Nachsicht bitten, wenn ich an verschiedenen Stellen auf die dortigen Darlegungen verweise. Für die Korrektur danke ich Lars Mojem. Teile des Beitrags stützen sich auf die Ausführungen in Knoblauch (2017).

2 Die Soziologie kennt dafür den Begriff der „Skripte“, die Handlungserwartungen werden als Rollen bezeichnet, und sie geht davon aus, dass körperliche Abläufe in feinsinnigster Weise sequentiell geordnet sein können, ohne dass die Sprache dabei eine Rolle spielen muss (Meyer/von Wedelstaedt 2017). 
„soziales Handeln“ ausschließt? Ist dann die „Außenstruktur“ etwas, das der Gattung äußerlich ist, sie nicht eigentlich betrifft? Und sind dann „soziale Strukturen“, wie etwa Verwandtschaft, Betriebe oder Staaten, unabhängig von kommunikativen Handlungen?

Wie andernorts ausgeführt (Knoblauch 2017), passt diese Unterscheidung nicht mehr in eine Gesellschaft, in der zunehmend nicht mehr nur die „weichen“ Wissensberufe mit Kommunikation ihr Geld verdienen und Kommunikation nicht nur Dinge durch die Welt jagt, sondern, etwa als Industrie 4.0, auch immer mehr zum Medium der Produktion materialer Güter dient. Kommunikation beschränkt sich hier nicht mehr auf eine von der Gesellschaftsstruktur unterschiedene „Kultur“, sie ist so wirkungsvoll geworden, dass sie nicht nur soziale Strukturen, sondern auch materiale Wirklichkeiten schafft.

Im Grunde ist dieser Gedanke schon in der ,gesellschaftlichen Konstruktion der Wirklichkeit“ angelegt, die Peter Berger und Thomas Luckmann (1969) ja ausdrücklich materialistisch verstanden wissen wollten. Um der Bedeutung der Kommunikation gerecht zu werden, reden wir deswegen auch von einer kommunikativen Konstruktion der Wirklichkeit. Der grundlegende Prozess, in dem diese Wirklichkeit konstruiert wird, ist deswegen auch mit dem Begriff des kommunikativen Handelns umschrieben. Im Unterschied zum sprachbezogenen „logozentrischen“ Begriff des kommunikativen Handelns bei Habermas (1981) schließt dessen kommunikativ konstruktivistische Version auch die Objektivierungen ein, die kraft des Körpers handelnd erzeugt werden müssen, um (als Teil reziproker sinnlicher Erfahrungen und Handlungen) überhaupt „sozial sichtbar“ zu sein. Bei diesen Objektivierungen kann es sich um den Körper und seine Bewegungen selbst handeln (etwa den Fingerzeig oder den Faustschlag), aber natürlich sind darin ebenso alle durch den Körper bewirkten Mediationen und die Technologien eingeschlossen. Erst auf dieser Grundlage können wir auch die gesellschaftliche Wirkkraft der Kommunikation erklären, die die gegenwärtige Kommunikationsgesellschaft auszeichnet.

Die Erweiterung vom sozialen zum kommunikativen Handeln wie auch dessen prinzipielle und gegenwärtige Bedeutung habe ich ebenso an anderer Stelle ausführlich erläutert (Knoblauch 2017). Hier möchte ich mich dagegen auf dessen Folgen für das Konzept der kommunikativen Gattung beschränken. Nach einer kurzen Erläuterung des Konzepts der kommunikativen Gattung möchte ich auf die erwähnten konzeptionellen Probleme hinweisen, die zum Begriff der kommunikativen Form führen. Im darauf folgenden Teil möchte ich dann den Begriff der kommunikativen Form erläutern, der in einem engen Zusammenhang mit der Wende zur kommunikativen Konstruktion steht: Wenn wir Kommunikation nicht auf sprachliches Handeln beschränken, sondern als verkörperten Vollzug ansehen, der nicht nur mit Zeichen, sondern auch mit anderen Objektivierun- 
gen, Dingen und Technologien verbunden ist, dann bedürfen wir auch einer Vorstellung der Fixierung und Institutionalisierung des kommunikativen Handelns. Das ist die kommunikative Form. Es handelt sich dabei um einen sozialtheoretischen Begriff, der ausdrücklich nicht auf die besonderen Fragen der Sprachwissenschaft ausgerichtet ist, sondern auf die gesamte Sozialwissenschaft. Wenn wir aber (noch) davon ausgehen dürfen, dass auch die Sprache nicht vom Himmel gefallen, sondern eine menschliche Leistung ist, und wenn wir auch davon ausgehen dürfen, dass sie nicht von einzelnen genialen Individuen, sondern Menschen im Zusammenspiel mit sich und ihrer materialen Wirklichkeit geschaffen bzw. konstitutiert wurde, dann ist auch die Linguistik im Grunde eine Sozialwissenschaft. Dies jedenfalls war eine der Grundannahmen für die Arbeit mit Susanne Günthner, und mit diesem Beitrag verbinde ich deswegen auch den Wunsch, dass diese Annahme eine der Grundlagen für die zukünftige Zusammenarbeit zwischen der Linguistik und der Soziologie bilden kann.

\section{Kommunikative Gattungen und ihre Probleme}

Ein Ziel der Analyse der kommunikativen Gattungen war die Frage, wie man die sequenzielle Ordnung von über kurze zwei- oder mehrgliedrige (konversationelle) Paarsequenzen hinausgehenden Sequenzen erfassen und erklären kann. Wie lässt sich die Ordnung dessen erklären, was nach dem Zeigen geschieht? Was geschieht, wenn die Frage beantwortet wurde, und warum wird sie gestellt? Denn auch längere Sequenzen sind zeitliche Prozesse: Die Frage, die in einer Seminarsitzung auf die Antwort trifft, ist ja etwas anderes als die Frage nach dem Weg. Selbst wenn Biografien umgekrempelt werden, wie etwa in Konversionen, verläuft auch diese Konversion ebenso wieder in besonderen Formen (Ulmer 1988).

Luckmann (1986) bezeichnet kommunikative Gattungen als verfestigte Formen kommunikativen Handelns. Verfestigt werden können dabei Objektivierungen, wie etwa „parasprachliche“ Merkmale (Prosodie, Rhythmus, Sprachgeschwindigkeit), sprachliche Mittel (Lexikon, Grammatik, Prosodie) und andere zeichenhafte Objektivationen (visuelle Motive, audiovisuelle Figuren u.a.). Verfestigt werden können auch sequenzielle Abläufe, wie etwa die Abfolge von Frage und Antwort, Befehl und Befehlserfüllung oder Blick und Gegenblick (etwa bei der Eröffnung des Flirtens). Solche Verfestigungen haben Keppler und Luckmann (1991) auch am Beispiel der informellen Belehrung aufgezeigt. In Interaktionen zwischen Gleichgestellten können solche Belehrungen kurzzeitig auftreten. Sie zeichnen sich durch dreigliedrige Sequenzen aus, in denen die Frage nicht nur eine Antwort einfordert, sondern diese auch eine Bewertung nach sich zieht 
(,gut“). Diese Belehrungen weisen eine besondere Sequenzialität auf, die das „Besserwissen“ schon in informellen Alltagsgesprächen für alle Beteiligten markiert (vgl. auch Couper-Kuhlen/Thompson i.d. B.). Der Verweis auf den sozialen Rahmen der Gespräche führt uns schon in die „Außenstruktur“ der kommunikativen Gattung, denn Belehrungen lassen sich auf verschiedenste Weisen institutionalisieren: In der Schule und anderen Einrichtungen der institutionalisierten Wissensvermittlung kann eine Frage mit Autorität gestellt werden, und wer antwortet, wird halbwegs dauerhaft definiert. Diese Sequenzen werden so mit besonderen Rollen verbunden, also etwa Lehrpersonal und Schülern, die mit verschiedenen Institutionen (Grundschule - Hochschule) oder Strukturen (Klassenunterschiede der Herkunft) variieren können (vgl. Kotthoff i. d. B. und Gülich i. d. B.).

So eindrücklich die bisherige Erforschung kommunikativer Gattungen ist, hat sie dennoch, wie schon erwähnt, einige theoretische Fragen aufgedeckt, die unbeantwortet geblieben sind.

a. Eine der offenen Fragen betrifft, erstens, das Verhältnis der kommunikativen Gattungen zu den Institutionen. So bezeichnet auch Luckmann (1986) die kommunikativen Gattungen als Verfestigungen und vergleicht sie darüber hinaus mit sozialen Institutionen. Kommunikative Gattungen sind ,wie“ Institutionen. Während Institutionen aus, richtigen ' sozialen Handlungen gebildet werden, bestehen die kommunikativen Gattungen aus seiner Sicht jedoch „,nur“ aus kommunikativen Handlungen, die für ihn eine Teilmenge sozialer Handlungen bilden. Deswegen sind kommunikative Gattungen Luckmann zufolge keine richtigen Institutionen. Offen bleibt deshalb, wie dann „Verfestigung“ zu verstehen sei, wenn es sich dabei nicht um eine Institutionalisierung handelt.

Weil wir uns hier auf die kommunikativen Formen beschränken, können wir das Problem der Verfestigung in diesem Beitrag nicht erläutern. Wir können nur andeuten, dass sich die Luckmannsche Unterscheidung so spezifizieren lässt, dass wir die Institutionalisierung von Handlungen, die Zeichen verwenden, von der Institutionalisierung nichtzeichenhaften Handelns unterscheiden müssen. Zeichen und zeichenhafte Objektivationen erfordern eine besondere Form der Institutionalisierung, die wir wohl besser mit dem Begriff der Konventionalisierung bezeichnen, den wir, in Anschluss an Luckmann, an anderer Stelle breiter ausgeführt haben (Knoblauch 2017: 252 ff.).

b. Es mag mit dieser Abgrenzung zu den ,richtigen' Institutionen zu tun haben, dass kommunikative Gattungen, zweitens, häufig eng auf stark formalisierte Muster (in Anlehnung an den üblichen Sprachgebrauch auch in der Volkskunde, der Literaturwissenschaft oder der Rhetorik als Gattung oder im engeren Sinn in der Linguistik als sprachliche Form bezeichnet) oder gar ästhetische Formen (Tanz, 
Gedicht etc.) beschränkt bleiben. Kommunikative Formen, die weniger verfestigt sind (wie etwa Konversationen), können mit diesem Begriff ebenso schwer erfasst werden wie hochgradig körperliche Performanzen, die keinen gerahmten Aufführungscharakter tragen (etwa beim Tanzen) (vgl. Stukenbrock i.d. B. und Bergmann/Peräkylä i.d. B.).

Aus diesen Gründen haben wir, wie schon erwähnt, vorgeschlagen, dem Begriff der Gattung die „kommunikativen Muster“ (,pattern“) an die Seite zu stellen, und auch der Begriff der Form ist dabei explizit verwendet worden (Knoblauch/ Günthner 1994, 1995). Muster unterscheiden sich von den Gattungen durch den Grad ihrer Verfestigung: Bilden etwa Notrufgespräche schon vor der Einführung digitaler Formate und unabhängig von schriftlichen Formularen Muster aus, so sind Predigten in christlichen Kirchen kommunikative Gattungen.

c. Drittens wurden kommunikative Gattungen bislang weitgehend am Beispiel sprachlichen kommunikativen Handelns konzipiert. Daher konzentrieren sich die meisten Analysen kommunikativer Gattungen auf Vollzüge mit starkem sprachlichem Anteil. Verfestigt werden etwa „parasprachliche“ Merkmale (Prosodie, Rhythmus, Sprachgeschwindigkeit), sprachliche Mittel (Lexikon, Grammatik, Prosodie) und andere zeichenhafte Objektivationen (visuelle Motive, audiovisuelle Figuren u. a.). Mit dem Einbezug der Interaktionsebene können auch die sequentiellen Muster als Verfestigungen betrachtet werden, doch bestehen diese sozusagen aus reinem Vollzug, wie er von Latour in seiner Kritik an der Ethnomethodologie formuliert wird. Ihre Materialität und Dinglichkeit werden ebenso wenig konzeptionell berücksichtigt wie die Körperlichkeit ihres Vollzugs (vgl. Stukenbrock i.d.B. und Deppermann i. d. B.). ${ }^{3}$

Diese Probleme des Gattungsbegriffs sind keineswegs nur theoretischer Natur. In meiner eigenen Untersuchung etwa von Powerpointpräsentationen (Knoblauch 2013) zeigen sie sich auch empirisch sehr konkret (vgl. auch Deppermann i.d. B. und Mazeland i. d. B. zu den Grenzen des Gattungsbegriffs). Während frühere Untersuchungen diese Gattung auf das Design der Folien reduziert hatten, öffnete der Begriff der kommunikativen Gattung den Blick auf die performative Seite dieser Gattung, also das Präsentieren durch menschliche Akteure. Dabei zeigte sich allerdings auch, wie sehr diese Gattung vom Foliendesign sowie der programmierten Serialität der Folien geprägt war. Vor allem aber zeigte sich, dass die technische und materielle Konstellation wesentlich für Powerpräsentationen ist: Die trianguläre Formation von Körper, Computer und Leinwand ist ein konstitutives

3 Man muss erwähnen, dass gerade die praxistheoretische Interpretation der Ethnomethodologie diese Verkürzung aufzufangen versucht, ohne jedoch je eine Integration der Gattungstheorie und ihres institutionalistischen Gedankens versucht zu haben. 
Merkmal für die Gattung und erklärt auch ihre wesentlichen Gattungsvariationen (also die körperbetonte performative, die Folienbezogene präsentierende und die quellenbezogene vorlesungsartige Präsentation).

Die Bedeutung der materiell-technischen Konstellation zeigte sich deutlich, doch wurde sie mir selbst durch den Begriff der Gattung verstellt. Die Präsentationssoftware, die digitale Verfügbarkeit von Folien und Folieninhalten sowie ihre Objektivierung, Archivierung und Kommodifizierung in Informations- und Wissensmanagementsystemen sind selbst Teil der Kommunikation und können damit auch zum verfestigten Kern kommunikativer Gattungen zählen und nicht nur zum sozialen Beiwerk. Der Begriff der kommunikativen Konstruktion würde es auch erlauben, ausschließlich körperliche Kommunikation als Gattung zu fassen; ebenso ließen sich Verfestigungen in den Blick nehmen, die ausschließlich in der Außenstruktur zu beobachten sind.

Der Begriff der kommunikativen Gattung führt aber auch in einer zweiten Weise leicht in die Irre. Denn auch wenn Powerpointpräsentationen deutlich längere Abfolgen von zumeist monologischen Sequenzen sind, so ist doch ihre Struktur enorm vielfältig. Sie kann sich ebenso an Vorlesungen wie an Festvorträgen anlehnen, kann aber auch die Form kurzer Verkaufsvorträge, „Pitches“, ja kleiner Instruktionen annehmen. Das ist einer der Gründe, die Powerpointpräsentation eher als eine Form zu bezeichnen denn als eine Gattung. Da sich dieser Begriff der Form auch für andere, ja vermutlich eine Vielfalt von empirischen Fällen nahelegt, ${ }^{4}$ stellt sich deswegen die Frage: Was verstehen wir unter dem Begriff der kommunikativen Form?

\section{Kommunikatives Handeln und kommunikative Formen}

Der Begriff der kommunikativen Form muss keineswegs von außen eingeführt werden, sondern findet sich schon in einem Aufsatz Luckmanns aus dem Jahre 1975, also deutlich vor seinen Überlegungen zu kommunikativen Gattungen. ${ }^{5}$ Im

4 Ein Beispiel dafür bietet die Form des „Grouptalk“, auf die wir im Rahmen der Untersuchung von Kooperationsformen in den interdisziplinären Neurowissenschaften gestoßen sind; sie lassen sich kaum als feste Gattung beschreiben, sondern nur durch allgemeine Kommunikationsregeln, wie sie vermutlich auch in anderen wissenschaftlichen und institutionellen Zusammenhängen auftreten. Vgl. Knoblauch/Lettkemann/Wilke (2017).

5 Den Begriff der kommunikativen Formen schlage ich andernorts als Oberbegriff für Gattungen und andere Verfestigungen kommunikativen Handelns vor (Knoblauch 2017: 217 ff.), doch ist mir 
Unterschied zu den „Sprachformen“, die Luckmann in derselben Zeit als elementare Konventionalisierungen in der Konstitution von Zeichen vorschlägt (Luckmann 1973), handelt es sich bei seinen „Kommunikationsformen“ um „Regelungen der kommunikativen Situationen“ (Luckmann 1975: 219 f.). ${ }^{6}$ Es geht ihm hier nicht um Sprache, sondern ihre Anwendung in sozialen Situationen (Luckmann 1975: 219). „In jeder Gesellschaft gibt es verschiedenartige kommunikative Situationen und demnach ein mehr oder minder reichhaltiges Angebot an Kommunikationsformen“ (Luckmann 1975: 220). Als Beispiele erwähnt er nicht nur Sprechakte, wie Fragen und Lieben, sondern auch Gespräche, Beratungen und Argumentationen, und er beschränkt sich nicht auf die Sprache, sondern bezieht auch Gestik, Körperhaltung oder Gesichtsausdruck mit ein. Dabei differenziert er die Kommunikationsformen nach dem, was man heute ihre „Modalität“ nennen würde (Hörsinn und besondere Lautformen, Gesichtssinn, Gestik etc.). Wie Gattungen sind diese Kommunikationsformen auch durch ihren „äußeren Ablauf“ bestimmt. „Dies gilt sowohl für die zwei wichtigsten Phasen kommunikativer Vorgänge, nämlich den Anfang und das Ende der Kommunikation und für die Regelung der ,Sprecherfolge‘, des ,Dranseins““ (Luckmann 1975: 219f). Wie er anschaulich ausführt, regeln die kommunikativen Formen auch, wer die Kommunikation beginnt, wie sie angefangen und beendet wird und wer beteiligt wird. Geregelt wird aber auch der „innere Verlauf“, also ihr Inhalt, die Mitteilungsabsichten und die Stilanforderungen.

Auch wenn wir Luckmanns Begriff der kommunikativen Formen aufnehmen, so müssen wir auf einen Unterschied - genauer: eine Erweiterung - hinweisen. Diese Ausweitung bezieht sich auf den Begriff des kommunikativen Handelns. Kommunikatives Handeln lässt sich zum einen nicht mehr als eine Art einseitiger, nur vom subjektiven Bewusstsein vorgenommener Entwurf fassen. Vielmehr betrachten wir ihn als eingebettet in eine reziprok angelegte Relation zwischen zwei Subjekten. Im Unterschied zu den meisten relationalen Ansätzen haben wir es hier aber nicht mit einer zweiwertigen flachen, sondern mit einer dreiwertigen triadischen Relation zu tun. Die Subjekte beziehen sich aufeinander, indem sie vermittels ihrer Körper Objektivierungen hervorbringen. Der Begriff der Objektivierung aber weist weit über die Sprache hinaus. Eine Objektivierung kann etwa der Finger sein, der anderen den Weg weist - eine zweifellos kommunikative Handlung; sie kann aber auch aus verdinglichten Objektivationen bestehen: statt

erst bei der Vorbereitung dieses Beitrags (wieder) eingefallen, dass dieses Konzept von Luckmann (1975) stammt.

6 Den Begriff der Konventionen, den ich auch aufnehme (Knoblauch 2017), betont Schütz in seinen Notizbüchern sehr ausdrücklich; Luckmann nimmt ihn dagegen nicht auf (Schütz/Luckmann 1984). 
des Fingers können wir den Stab verwenden, und wenn wir technisch raffiniert werden, den „Laserpointer“. ${ }^{7}$ Mit der Objektivierung kommt die Körperlichkeit in den Begriff des kommunikativen Handelns, die es performativ macht. ${ }^{8}$ Für den Begriff der kommunikativen Form aber folgt daraus, dass er nicht der gängigen aristotelischen Unterscheidung zur Materie folgt. Form ist nicht, wie Aristoteles meint, dasjenige, was zur Materie hinzutritt, um etwas zu sein.

Die Materie kann natürlich material zu einer Gestalt gerinnen, wie dies etwa in der Architektur mustergültig geschieht; sie ist aber auch Teil des kommunikativen Handelns; sei es im Wirken des Körpers (das sich nicht aufs Boxen beschränkt); es tritt aber auch in der sinnlich wahrnehmbaren Stimme, im handgeschriebenen Schriftzeichen oder im getippten Smiley auf, und zwar in einer Weise, die keineswegs nur beiläufig ist, sondern den Sinn durch seine Sinnlichkeit bestimmt.

Es ist die Objektivierung, die dem Prozess der Verfestigung etwas hinzufügt, das Latour (2005) an den bisherigen Konzepten des Sozialen vermisst: Die Verfestigung ist nicht ein flüchtiges Geschehen, das sich lediglich je im immateriellen Sinn manifestiert. Die Objektivation verleiht dem Handeln eine Stabilität, die über den schieren Augenblick hinausreicht; sie ist die Grundlage dessen, was wir als die Wirklichkeit des Sozialen ansehen.

Die im Rahmen des kommunikativen Konstruktivismus vollzogene Ausweitung des kommunikativen Handelns auf alle sozialen Handlungen hat jedoch Folgen für das Konzept der kommunikativen Formen. Diese begriffliche Ausweitung hat zum einen zur Folge, dass kommunikative Formen auch durch körperliche, dingliche und materiale Aspekte des kommunikativen Handelns gebildet werden. Kommunikative Formen lassen sich nicht nur durch verfestigte sprachliche Abläufe bestimmen. Sie lassen sich als verfestigte körperlich-performative Abläufe, als performative Gattungen beschreiben. Erst durch eine solche Ausweitung können wir auch Powerpointpräsentationen erfassen, aber auch Arztkonsultationen oder gar Massenereignisse, wie wir sie am Beispiel der Papstmesse im Berliner Olympiastadion 2011 untersucht haben (Knoblauch 2011). Diese Ausweitung bedeutet, dass wir die materiale Anordnung und die Formation der Körper in die Betrachtung der kommunikativen Form mit einbeziehen, etwa die räumliche Ar-

7 Wenn man hier ein institutionell hochspezifisches Beispiel eines solchen Designs heranziehen kann, dann ist es der Zuschnitt des Hammerschlags von Auktionatoren auf den Ablauf von Geboten, wie er von Heath (2013) bis ins kleinste Detail als interaktiv koordiniert (und dabei strategisch geleitet) analysiert wurde.

8 Wie andernorts ausgeführt (Knoblauch 2017: 155 ff.) bezeichne ich Objektivierungen als jene Kommunikationsmedien, die mit dem Körper verbunden (Zeigefinger) sind, während Objektivationen als vom Körper als abgelöst betrachtet werden (Zeigestab, gemalter Pfeil). 
chitektur des Petersplatzes oder von Stadien und Hallen. Auch die Technologien und Kommunikationsmedien sind wesentlich, wie etwa die akustischen Systeme, ohne die solche Großveranstaltungen in dieser Form gar nicht stattfinden könnten. Natürlich ist auch die soziale Struktur, die sich etwa in der eventorganisatorisch vorbereiteten räumlichen Aufteilung im Stadion niederschlägt, kein externes Merkmal, sondern durch die verschiedenen Partizipationsweisen des Publikums ebenso Teil der Form.

Kommunikative Formen bestehen aus synchron und diachron, räumlich und zeitlich ausgeweiteten Sequenzen. Während die kommunikative Handlung auf eine lediglich analytische Weise den Prozess beschreibt, aus dem das Soziale besteht, stellt die Sequenz des kommunikativen Handelns und ihre Einheit (die von einer monologischen über eine „Paarsequenz“ bis zu langen Sequenzfolgen reichen kann) schon eine entschieden empirische Größe dar. Dass soziales Handeln empirisch immer kommunikativ ist, zeigt sich frühestens in der Sequenz. Sequenzen kommunikativen Handelns, wie etwa Frage und Antwort, Grußgeste und Gegengruß (vgl. Linke i. d. B.), Zeigen und Blicken (sowie deren Vereinseitigungen), weisen zwar allgemeine Gemeinsamkeiten auf, ${ }^{9}$ doch nehmen sie empirisch auch höchst unterschiedliche und vielfältige Formen an. Dies gilt noch für die Fortsetzung über diese elementare Paarsequenz hinaus (vgl. auch Mazeland i.d. B.). Sequenzen sind durch das kommunikative Handeln in nur so groben Zügen festgelegt, dass diese Fortsetzungen hochgradig kontingent sind. Bei jedem weiteren Zug stellt sich die Frage, wie fortgesetzt werden kann (auch und gerade, wenn die Sequenz abgeschlossen scheint). Die Frage der sequenziellen Fortsetzbarkeit ist, wie die Einheit des Handelns, thematisch offen. Sie wird empirisch durch die Verwendung eingespielter Formen gelöst. Kommunikative Formen sind der Grund dafür, dass Gesellschaften keine gestaltlosen Gebilde sind, und zwar auch dort, wo sie nicht materialisiert sind und wo sie keine ausgeprägten sozialen Morphologien oder „Strukturen“ aufweisen. Kommunikative Formen sind Abläufe kommunikativer Sequenzen mit einer gewissen Kontur, also mit einem Anfang und ein Ende aufweisen. Deswegen sollte der Begriff der Form von seiner gängigen aristotelischen Bedeutung (Aristoteles 1995) abgegrenzt werden, die sie von der Materie unterscheidet. Form ist nicht aristotelisch dasjenige, was zur Materie hinzutritt, um etwas zu sein. Im Unterschied zu dieser substanzialistischen Vorstellung der Form geht es uns um einen prozessualen Begriff der Form, der sich auf das kommunikative Handeln und die aus ihm gebildeten Sequenzen bezieht.

In der Soziologie wurde die prozessuale Vorstellung der Form vor allem von Simmel geprägt. Denn Simmel (1992) hebt mit der „sozialen Form“ die Eigenstän-

9 Goffman (1978) hat sie als „Aufforderungen“ und „Erwiderungen“ bezeichnet. 
digkeit des Sozialen hervor, die auch der Begriff der kommunikativen Form anspricht. ${ }^{10}$ Simmel bezeichnet als Form bestimmte Arten von sozialen Wechselwirkungen, wie etwa Geselligkeit, Konkurrenz, Parteibildung, Über- und Unterordnung, Nachahmung, Arbeitsteilung, Vertretung. Bevor in seinem späteren Werk die Bedeutung des Statischen und Erstarrten, also das, was später Struktur heißt, in den Vordergrund tritt, behandelt er die Form entschieden als einen Gegenbegriff zum Inhalt: Die Konkurrenz als Form etwa kann am Beispiel des „Marktes“ mit wirtschaftlichen Inhalten auftreten, sie kann aber auch sportlich als „Wettkampf“ gefüllt werden. So eingängig diese Unterscheidung zwischen Form und Inhalt ist, führt sie doch in eine „formale“ Soziologie, die die Sozialwelt in zwei Reiche aufteilt: Inhaltliche Kultur und formale Struktur treten auseinander, ohne dass sich eine Verbindung herstellen ließe.

Eine besondere Lösung für diese Zweiteilung bietet die Ethnomethodologie. Denn die schon erwähnten „Ethnomethoden“ sind ja (ebenso wie das kommunikative Handeln) nicht nur die Antwort auf die Frage, wie soziale Ordnung möglich ist. Wie alle Methoden bieten diese Ethnomethoden eine Antwort auf die sozialkonstruktivistische Frage: Wie wird die soziale Wirklichkeit erzeugt? Und gerade im „Wie“ liegt auch ihre Antwort: Indem wir auf bestimmte Weisen handeln, erzeugen wir eine Art der Ordnung. Die soziale Wirklichkeit „ist“ nicht, sondern besteht in der Methode, wie sie erzeugt wird. Das „Was“, die vermeintliche Substanz, wird im „Wie“ des Prozesses erzeugt. Auch wenn die Ethnomethodologie den Begriff nicht hervorhebt, so liegt es nahe: Die Antwort auf die Frage nach dem Wie ist die Form. ${ }^{11}$

Im Unterschied zu Simmel stellt die Ethnomethodologie die Form dem Inhalt allerdings nicht gegenüber, sondern betont, dass die Form den Inhalt bestimmt: Was wir machen, ist ganz wesentlich geleitet von dem, wie wir es tun. Predigten unterscheiden sich durch besondere Formen von politischen Reden, Arztkonsultationen zeichnen sich dadurch aus, wie sie durchgeführt werden, nämlich in Sprechstunden, und in Verkaufsshows wird anders gehandelt als in wissenschaftlichen Präsentationen (wobei auch die Übernahme und Angleichung von Formen soziologisch höchst aussagekräftig sein kann). Dabei sind die Ethnomethoden nicht einfach kontingent, sondern durch ihre zeitliche Verkörperung immer in einer minimalen, aber materialen Weise spezifiziert: Was macht eine Frage

10 Wie Rothenbühler (2009: 287) zeigt, gibt es diese kommunikative Form auch bei Bühler; sie setzt sich aus einem ,element of the internal world“, dem ,externalized, given material“ und „the other“ zusammen. Diese Triade kennzeichnet auch für uns das kommunikative Handeln, genügt aber noch nicht selbst als Formbestimmung.

11 Dabei scheint die Form eher eine außenstehende Beschreibungskategorie zu sein, die Methode dagegen eine praktische Kategorie bei der Performanz. 
zur Frage, was einen Streit zum Streit, was einen Mann zum Mann oder eine Frau zur Frau?

Die Sequenz, die Situation, das Geschlecht, was immer sozial wirklich sein mag, geht also darauf zurück, wie etwas konstruiert, hergestellt, fabriziert oder produziert wurde, mit welchen Methoden es erzeugt wurde und welche kommunikativen Handlungen vollzogen wurden.

Das „Wie“ der Ethnomethodologie ist zweifellos ein zentraler Aspekt der kommunikativen Form. Zugleich wirft jedoch der Situationalismus der Ethnomethodologie große Probleme auf: Die soziale Wirklichkeit gilt der Ethnomethodologie als eine „Vollzugswirklichkeit“ (Bergmann 1981), die ständig im Fluss ist und in jeder Situation wieder neu geordnet werden muss. Dieser situative Konstruktivismus wirft die Frage auf, wie denn die Ethnomethodologie überhaupt Gesellschaft als etwas, das das Situative der Interaktion überschreitet, fassen kann (KnorrCetina 1981).

Bei der Suche nach einer Antwort auf diese Frage werden wir fündig bei dem, was Baecker im Gefolge von Luhmann als „Form“ bestimmt. Dieser systemtheoretische Begriff der Form erlaubt es, „eine Unterscheidung als Zusammenhang des Unterschiedenen zu beobachten“. Die Form zeichnet also nicht nur das aus, was sie selbst ist, sondern unterscheidet es nach außen. Die Form schließt das ein, was sie ausschließt, lautet die entsprechend etwas paradoxe Formulierung Baeckers (2005: 68). Die Form ordnet nicht nur nach innen; sie ist auch nach außen als Form erkennbar.

Diese Leistung kann auch in unserer Begrifflichkeit durch eine schon erwähnte Eigenschaft des kommunikativen Handelns erklärt werden, nämlich die kommunikative Reflexivität:12 Kommunikative Formen verdanken sich vor allem der Reziprozität des kommunikativen Handelns. Sie zeichnen sich dadurch aus, dass sie nicht nur auf eine Weise erzeugt werden, sondern dass sie als so erzeugt reziprok auch für andere wahrnehmbar (systemtheoretisch: „beobachtbar“) gemacht werden. Die Leistung der Form ist, dass sie etwas erkennbar macht, was ohne sie nicht erkennbar wäre. Die kommunikative Form ist zwar nicht notwendig selbst eine Objektivierung, wird aber an Objektivierungen erkennbar gemacht. Sie ist reflexiv, weil sie nicht nur eine Form ist, sondern die Form die Differenz und Ähnlichkeiten $\mathrm{zu}$ anderen Formen reflektiert und damit auch einen Hinweis darauf gibt, wie sie selber vollzogen und verstanden werden kann. ${ }^{13}$

12 Um die Erkennbarkeit nach außen zu erklären, müssten wir vermutlich auf die Figur des Dritten zurückgreifen, die ja die Sequenz als Ganze erfassen muss. Allerdings muss die Form auch nach innen, also von den Beteiligten selbst erkannt werden.

13 Dass kommunikatives Handeln insbesondere in seiner Sequenzialität reflexiv ist, haben wir oben ja schon ausgeführt. Dies hat im Wesentlichen zur Folge, dass es nicht nur performativ 
Ihre Reflexivität drückt sich schon in den Gestalten der Objektivationen aus, wie etwa dem schon erwähnten „Zeigefinger“, dem „Zeiger“, dem „Pfeil“ oder dem „Laserpointer“. Wie die Frage nicht nur an der Antwort, sondern an der Frage-Antwort-Sequenz orientiert ist, so ist die Begrüßung nicht nur auf den Gegengruß gerichtet, sondern an einer (zumeist auch komplexeren) Ablaufform, der Begrüßung, orientiert, die auch als Ritual konventionalisiert werden kann. Das gilt auch für die nichtsprachlichen Abläufe, wie das freundliche Grinsen beim gegenseitigen Erkennen, das vom zwischenzeitlichen Wegblicken (gerne nach unten) gefolgt wird, während man aufeinander zuläuft, um beim Aufeinanderzugehen in kurzer Distanz wieder mit einem Lachgesicht aufzublicken.

Die Form ist also mehr als eine kommunikative Handlung, sie ist auch mehr als eine Sequenz. Wenn sie nicht schon eine konventionalisierte Objektivation ist (wie das Pfeilzeichen), dann ist sie im performativen Vollzug zumindest eine koproduzierte Objektivierung. Weil sie als Form wahrgenommen werden kann, erlaubt sie damit die sequenzübergreifende Durchführung der kommunikativen Handlungen. Als Form dient sie nicht nur zur Orientierung von Handlungen der daran Beteiligten nach innen, sie kann aber auch nach außen unterschieden werden: So nehmen Anrufungen der Götter (auch der Jagdgötter) eine andere Form an als Jagdrufe, die zur Koordination von Jägern dienen; politische Versammlungen nehmen eine andere Form an als religiöse (sofern eben Religion von der Politik unterschieden wird) und Eide eine andere als Versprechungen.

Die Form kann sich durch einen gemeinsamen Stil auszeichnen - denken wir nur an das Tanzen, das als Tanzen eigene Formen körperlicher Performanzen ausbildet. Bei längeren, wiederkehrenden oder institutionalisierten Sequenzen kann die Form daneben auch besondere Markierungen, Codes oder Rahmen des kommunikativen Handelns annehmen. Diese Stile, Markierungen, Codes oder Rahmungen müssen aber nicht unbedingt eine ganze und vollständige Gattung definieren („Es war einmal“ und das Märchen), sondern können auch als einzelne Aspekte der Form verfestigt sein und etwa nur einen spielerischen (Theatervorhang) oder performativen Modus anzeigen. Allerdings haben wir es bei Markierungen, Codes und Rahmungen schon mit besonderen konventionalisierten Formen zu tun.

Formen müssen sich nicht auf der Ebene der Zeichen verfestigen. Sie können sich auch durch Veranstaltungsarten (wie etwa Sportspiele in Stadien oder Events), Institutionen oder ganze Institutionsbereiche auszeichnen. So weisen etwa schulische Einrichtungen und Institutionen eine besondere Form auf, die Thé-

durchgeführt wird, sondern zugleich anzeigt, wie es zu verstehen sein soll (zumeist in einem zeitlich elementar sequenziellen Prozess, der ebenso schon skizziert wurde). 
venaz-Christen (2005) als „forme scolaire“ bezeichnet. Sie charakterisiert schulische Einrichtungen verschiedenster Art (von den Schulstunden über Schulgebäude bis zu bestimmten Geräten) und greift damit weit über das hinaus, was die Systemtheorie als „Codes“ in diesem Fall: der Bildung bezeichnet. Doch wie wir mit den Codes die Ebene der konventionalisierten Zeichen betreten, befinden wir uns mit dem Beispiel der Bildung schon im Bereich der sozialen Strukturen und Institutionen (vgl. Kotthoff i.d. B.).

Hervorzuheben ist, dass die Formen, auch wenn sie unter anderem der Unterscheidung dienen, keineswegs binären Mustern folgen oder scharf voneinander abgrenzbar sein müssen. Zwar können manche Gesellschaften beispielsweise das Religiöse und seine kommunikativen Formen säuberlich vom Politischen unterscheiden, indem sie etwa die entsprechenden Abläufe (Beten und Herrschen), Personen (Schamanen und Häuptlinge) oder Räume (Kirchen und Parlamente) scharf auseinanderhalten; von einer Form aber können wir auch reden, wenn diese Unterscheidungen nicht vorgenommen werden: Ob ein Ritual zugleich der Verehrung der Götter, dem Zusammenhalt der Gemeinschaft oder dem Genussstreben der daran Beteiligten dient, ändert nichts daran, dass es sich um eine klare Form handeln kann. ${ }^{14}$ Wenn wir wissen, wie etwas getan wird oder dass es „so“ geht, müssen wir keineswegs notwendig auch die anderen Formen kennen. Kommunikative Formen sind also noch nicht notwendig Gattungen, sie weisen auch nicht Strukturen auf, in denen Elemente eindeutig isoliert sind. ${ }^{15}$

\section{Kommunikative Formen, kommunikative Gattungen und die Kommunikationsgesellschaft}

Nachdem wir ausgeführt haben, was kommunikative Formen sind, stellt sich die Frage, wie Formen und Gattungen zueinander stehen. Es sollte deutlich geworden sein, dass der Begriff der kommunikativen Form nicht eine Alternative zur kommunikativen Gattung darstellt. Es handelt sich vielmehr um einen grundlegenderen Begriff: Kommunikative Gattungen sind kommunikative Formen, aber

14 Ein schon etwas elaboriertes Beispiel dafür ist eine schamanistische Zeremonie in einer Gesellschaft, die Schamanen nicht als Rollen ausdifferenziert hat; hier kann das, was in anderen Gesellschaften als technisches, als religiöses, als medizinisches Handeln etc. ausdifferenziert ist, ununterschieden miteinander verknüpft sein.

15 Cassirer (1990) verwendet dazu den Begriff der symbolischen Formen, der die verschiedenen Symbolbereiche von Wissenschaft, Kunst oder Religion charakterisiert und voneinander abgrenzt. 
kommunikative Formen sind keineswegs notwendig kommunikative Gattungen. Kommunikative Formen zeichnen sich zum einen durch eine geringere Verfestigung aus sowie durch einen geringeren Grad an Institutionalisierung. Dieser Grad ist übrigens soziologisch keineswegs metaphorisch. Vielmehr sind Gattungen als kommunikative Institutionen auch mit bestimmten Normen verknüpft, deren Einhaltung geregelt wird; die Abweichung von diesen Normen wird zwar selten unter Strafe gestellt, so dass es sich zumeist um „Kann-Normen“ handelt; wer allerdings vor Gericht nicht in der Lage ist, eine Zeugenaussage zu machen, wer in der mündlichen Prüfung die Form des Prüfungsgespräches nicht beherrscht - vom Bewerbungsgespräch ganz zu schweigen -, wird bemerken, dass es hier durchaus auch härtere „Soll-“ und „Muss-Normen“ gibt, die mit erkennbaren Sanktionen verbunden sind. Wie wir etwa aus der schulischen Kommunikation wissen, kann der Mangel an solchen kommunikativen Kompetenzen massive sozialstrukturelle Konsequenzen haben und zum Ausschluss ganzer sozialer Klassen oder, wie die PISA-Untersuchungen andeuten, ethnischer Gruppen führen.

Diese offenkundige Verbindung zur Sozialstruktur aber wirft auch die Frage auf, wie wir ihr Verhältnis zu kommunikativen Gattungen fassen. Dabei sehe ich zwei Möglichkeiten: Zum einen können wir den Begriff der Gattung ausweiten, so dass er die materialen und sozialstrukturellen Elemente mit einschließt. Diese Ausweitung würde parallel zur Ausweitung des Handlungsbegriffes verlaufen, würde aber auch zu einer größeren Unschärfe des Begriffes führen. Denn in diesem Falle wären auch Veranstaltungstypen, Organisationsformen und soziale Strukturen, wie etwa Milieus oder Klassen, im Konzept der kommunikativen Gattungen enthalten, so dass etwa Konversationsanalysen lediglich einen minimalen Beitrag zur Bestimmung von Gattungen erbringen könnten. Es scheint mir deswegen sinnvoller, den bisherigen Begriff der Gattung beizubehalten, der sich ja für die Analyse verfestigter Formen sprachlich vollzogener Kommunikation durchaus bewährt hat. Folgen wir dieser begrenzten Fassung, dann müssen wir allerdings auch das analytische Konzept der kommunikativen Gattungen einem breiteren Konzept unterstellen, unter dem wir andere Arten der Kommunikation fassen können. Dieser Begriff ist, wie vorgeschlagen, die kommunikative Form. Weil er sowohl körperlich-sinnliche, materiale wie auch technologisch-dingliche Objektivierungen einschließt, kann er sich auch auf körperliche Interaktionen beziehen, wie etwa Boxkämpfe. Dabei kann es sich um sehr stark institutionalisierte Formen handeln, wie etwa Handballspiele; es können aber auch Liebesakte sein, die bislang zwar in ihrer „geskripteten Form“, interessanterweise aber kaum als kommunikative Formen mit ihrer besonderen Körperlichkeit und Sinnlichkeit betrachtet wurden. (Ähnliches gilt auch für Gewalt.) Dass technische Medien mit ihrer besonderen „Affordanz“ eigene Verfestigungen ausbilden, die sich auch in den technischen Kommunikationsmedien niederschlagen (etwa im Unterschied zwi- 
schen dem Lehrbuch und dem Video-Tutorial), und dass Objektivationen auch als Objekte und Materialitäten kommunikative Formen annehmen (als Gestalt, Design oder Architektur), folgt der Alltagssemantik des Begriffes Form. Sie deuten aber auch an, wie sich die kommunikativen Formen auf die vermeintlich „makrostrukturelle" Ebene erstrecken: Techniken sind unmittelbar mit Infrastrukturen verbunden, Objekte und Architekturen mit Räumen, Verbindungen und Grenzen, die durch ihre Verfestigungen soziale Strukturen bilden: Privaträume, nationale Kabelnetzwerke, Städte oder Warenzirkulationen.

Indem der Begriff der kommunikativen Form an dem weiteren Begriff des kommunikativen Handelns anschließt, erlaubt er es auch, körperliche, materiale und damit immer auch soziale Wirkzusammenhänge zu erfassen, die in der Kommunikation erzeugt werden. Kommunikatives Handeln beschränkt sich eben nicht auf „reine soziale Beziehungen“, sondern schafft die gesellschaftliche Wirklichkeit. Dieser materialistisch-konstruktive Aspekt des kommunikativen Handelns tritt gerade in den letzten Jahrzehnten sehr deutlich zutage. Die Digitalisierung technischer Systeme bedeutet ja eine Verbindung materieller Zusammenhänge mit binär-zeichenhaften Regelsystemen, die von Menschen so erzeugt und programmiert werden, dass sie relativ eigenständig operieren (,intraaktiv“) oder die an menschliche Handelnde auf eine Weise angekoppelt werden, dass wir von Mediatisierung reden müssen. Diese digitale Mediatisierung ermöglicht die Einsicht in den kommunikativen Charakter der gesellschaftlichen Konstruktion, weil sie die gegenwärtige Wirklichkeit in einer unübersehbaren Weise hervorbringt: Kommunikatives Handeln generiert fortwährend Daten, die als Objektivationen gespeichert und wieder abgerufen, genutzt und neu bearbeitet werden können; kommunikatives Handeln bildet auch die Arbeit, die wir verrichten, und zwar nicht nur für die schreibenden Akademiker, sondern auch für die digital vernetzten EU-Bauern; und kommunikatives Handeln wird zunehmend sogar in der „alten“ Industrieproduktion sichtbar, die sich zur „Industrie 4.0“ wandelt. Wenn wir diesen sozialen Wandel als „Kommunikationsgesellschaft“ bezeichnen, dann geht es nicht mehr nur um eine neue gesellschaftliche Ordnung; der Begriff der kommunikativen Form erlaubt uns vielmehr auch die Beobachtung einer Ordnungsbildung, die nicht auf die Strukturen fixiert bleibt, sondern den Wandel selbst erfassen kann. ${ }^{16}$

16 Um diese Art des Wandels zu bezeichnen, habe ich den Begriff der Re-Figuration vorgeschlagen, der an Elias' Kritik des Strukturbegriffes orientiert ist. 


\section{Literatur}

Aristoteles (1995): Metaphysik. Hamburg: Meiner.

Baecker, Dirk (2005): Form und Formen der Kommunikation. Frankfurt a. Main: Suhrkamp.

Berger, Peter L. und Thomas Luckmann (1969): Die gesellschaftliche Konstruktion der Wirklichkeit. Frankfurt a. Main: Fischer.

Bergmann, Jörg R. (1981): Ethnomethodologische Konversationsanalyse. In: Schröder, Peter und Hugo Steger (Hrsg.), Dialogforschung. Jahrbuch des Instituts für deutsche Sprache, S. 9-51. Düsseldorf: Schwann.

Bergmann, Jörg und Anssi Peräkylä (i. d. B.): Traumdarstellungen als Narratoid. Epistemische Sprünge bei der Wiedergabe von Träumen. In: Weidner, Beate et al. (Hrsg.), Verfestigungen in der Interaktion. Konstruktionen, sequenzielle Muster, kommunikative Gattungen. Berlin: de Gruyter.

Cassirer, Ernst (1990): Versuch über den Menschen. Einführung in eine Philosophie der Kultur. Frankfurt a. Main: Fischer.

Couper-Kuhlen, Elizabeth und Sandra A. Thompson (i. d. B.): Ratschläge in der Alltagskommunikation: Zur Verwendung einer sedimentierten Form im Englischen. In: Weidner, Beate et al. (Hrsg.), Verfestigungen in der Interaktion. Konstruktionen, sequenzielle Muster, kommunikative Gattungen. Berlin: de Gruyter.

Deppermann, Arnulf (i. d. B.): Imperative im Deutschen: Konstruktionen, Praktiken oder social action formats? In: Weidner, Beate et al. (Hrsg.), Verfestigungen in der Interaktion. Konstruktionen, sequenzielle Muster, kommunikative Gattungen. Berlin: de Gruyter.

Goffman, Erving (1978): Erwiderungen und Reaktionen. In: Kölner Zeitschrift für Soziologie und Sozialpsychologie, Sonderheft, 20: Materialien zur Soziologie des Alltags:120-176.

Gülich, Elisabeth (in Zusammenarbeit mit Martin Schöndienst) (i. d. B.): „Ich dachte, Sie stellen Fragen." Irritationen und Aushandlungsprozesse im Zusammenhang mit Gattungserwartungen. In: Weidner, Beate et al. (Hrsg.), Verfestigungen in der Interaktion. Konstruktionen, sequenzielle Muster, kommunikative Gattungen. Berlin: de Gruyter.

Günthner, Susanne (2000): Vorwurfsaktivitäten in der Alltagsinteraktion. Grammatische, prosodische, rhetorisch-stilistische und interaktive Verfahren bei der Konstitution kommunikativer Muster und Gattungen. Tübingen: Niemeyer.

Günthner, Susanne (2006): Grammatische Analysen der kommunikativen Praxis - ,Dichte Konstruktionen' in der Interaktion. In: Deppermann, Arnulf, Reinhard Fiehler und Thomas Spranz-Fogasy (Hrsg.), Grammatik und Interaktion - Untersuchungen zum Zusammenhang von grammatischen Strukturen und Gesprächsprozessen, S. 95-122. Radolfzell: Verlag für Gesprächsforschung.

Günthner, Susanne und Helga Kotthoff (1991): Von fremden Stimmen. Weibliches und männliches Sprechen im Kulturvergleich. Frankfurt a. Main: Suhrkamp.

Günthner, Susanne, Dagmar Hüpper und Constanze Spieß (Hrsg.) (2012): Genderlinguistik. Sprachliche Konstruktionen von Geschlechtsidentität. Berlin: de Gruyter.

Günthner, Susanne und Hubert Knoblauch (1994): „Forms are the Food of Faith“. Gattungen als Muster kommunikativen Handelns. In: Kölner Zeitschrift für Soziologie und Sozialpsychologie, 4:693-723.

Günthner, Susanne und Hubert Knoblauch (1995): Culturally patterned speaking practices - the analysis of communicative genres. In: Pragmatics, 5:1-32. 
Günthner, Susanne und Hubert Knoblauch (1996): Die Analyse kommunikativer Gattungen in Alltagsinteraktionen. In: Michaelis, Susanne und Doris Tophinke (Hrsg.), Texte - Konstitution, Verarbeitung, Typik, S. 35-57. München: Lincom Europa.

Günthner, Susanne und Hubert Knoblauch (2000): Textlinguistik und Sozialwissenschaften. In: Brinker, Klaus et al. (Hrsg.), Text und Gesprächslinguistik. Linguistics of Text and Conversation, 1. Halbband, S. 811-819. Berlin: de Gruyter.

Günthner, Susanne und Hubert Knoblauch (2007): Wissenschaftliche Diskursgattungen Powerpoint et al. In: Auer, Peter und Harald Baßler (Hrsg.), Reden und Schreiben in der Wissenschaft, S. 53-66. Frankfurt a. Main: Campus.

Habermas, Jürgen (1981): Theorie des Kommunikativen Handelns. 2 Bände. Frankfurt a. Main: Suhrkamp.

Heath, Christian (2013): The Dynamics of Auction: Social Interaction and the Sale of Fine Art and Antiques. Cambridge: CUP.

Keppler, Angela und Thomas Luckmann (1991): „Teaching“: Conversational Transmission of Knowledge. In: Markova, Ivana und Klaus Foppa (Hrsg.), Asymmetries in Dialogue, S. 143-165. Hertfordshire: Harvester \& Wheatsheaf.

Knoblauch, Hubert (1990): The taming of foes. Informal Discussions in Family Talk. In: Markova, Ivana und Klaus Foppa (Hrsg.), Asymmetries in Dialogue, S. 166-194. New York: Harvester \& Wheatsheaf.

Knoblauch, Hubert (2011): Videoanalyse, Videointeraktionsanalyse und Videographie - zur Klärung einiger Mißverständnisse. In: sozialer sinn, 1:139-147.

Knoblauch, Hubert (2013): Powerpoint, Communication, and the Knowledge Society. New York: CUP.

Knoblauch, Hubert (2017): Die kommunikative Konstruktion der Wirklichkeit. Wiesbaden: VS.

Knorr-Cetina, Karin (1981): The Micro-Sociological Challenge of Macro-Sociology. In: KnorrCetina, Karin und Aaron V. Cicourel (Hrsg.), Advances in Social Theory and Methodology: Toward an Integration of Micro- and Macro-Sociologies, S. 1-47. Boston: Routledge \& Kegan Paul.

Kotthoff, Helga (i. d. B.): Zum Indizieren schulorientierter Mutterschaft in Lehrperson-ElternGesprächen. In: Weidner, Beate et al. (Hrsg.), Verfestigungen in der Interaktion. Konstruktionen, sequenzielle Muster, kommunikative Gattungen. Berlin: de Gruyter.

Latour, Bruno (2005): Reassembling the Social: An Introduction to Actor-Network-Theory. Oxford: OUP.

Linke, Angelika (i. d. B.): Musterwandel als Indikator für soziokulturellen Wandel. Ein Abriss zur Veränderung von Grussformeln vom 17. zum 21. Jahrhundert. In: Weidner, Beate et al. (Hrsg.), Verfestigungen in der Interaktion. Konstruktionen, sequenzielle Muster, kommunikative Gattungen. Berlin: de Gruyter.

Luckmann, Thomas (1973): Aspekte einer Theorie der Sozialkommunikation. In: Althaus, Hans Peter, Helmut Henne und Herbert Ernst Wiegand (Hrsg.), Lexikon der Germanistischen Linguistik, S. 1-13. Tübingen: Niemeyer.

Luckmann, Thomas (1975): Kommunikation, das Individuum und die Gesellschaft. In: Reden und reden lassen, S. 213-229. Stuttgart: Deutsche Verlags Anstalt.

Luckmann, Thomas (1986): Grundformen der gesellschaftlichen Vermittlung des Wissens: Kommunikative Gattungen. In: Kölner Zeitschrift für Soziologie und Sozialpsychologie, Sonderheft, 27: Kultur und Gesellschaft:191-211. 
Mazeland, Harrie (i. d. B.): Die Aktivität als eigenständiger interaktionsorganisierender Bereich. In: Weidner, Beate et al. (Hrsg.), Verfestigungen in der Interaktion. Konstruktionen, sequenzielle Muster, kommunikative Gattungen. Berlin: de Gruyter.

Meyer, Christian und Ulrich von Wedelstaedt (2017): Intercorporeality, interkinaesthesia, and enaction: new perspectives on moving bodies in interaction. In: Meyer, Christian und UIrich von Wedelstaedt (Hrsg.), Moving Bodies in Interaction - Interacting Bodies in Motion, S. 1-27. Amsterdam: Benjamins.

Rothenbühler, Eric W. (2009): Continuities: Communicative Form and Institutionalization. In: Lundby, Knut (Hrsg.), Mediatization: Concept, Changes, Consequences, S. 277-292. New York: Peter Lang.

Schütz, Alfred und Thomas Luckmann (1984): Strukturen der Lebenswelt, Band II. Frankfurt a. Main: Suhrkamp.

Simmel, Georg (1992): Soziologie: Untersuchungen über die Formen der Vergesellschaftung. Frankfurt a. Main: Suhrkamp.

Stukenbrock, Anja (i. d. B.): Mit Blick auf die Geste - multimodale Verfestigungen in der Interaktion. In: Weidner, Beate et al. (Hrsg.), Verfestigungen in der Interaktion. Konstruktionen, sequenzielle Muster, kommunikative Gattungen. Berlin: de Gruyter.

Thévenaz-Christen, Therese (2005): Les prémices de la forme scolaire: études d'activités langagières orales à l'école enfantine genevoise. Dissertation, Universität Genf.

Ulmer, Bernd (1988): Konversionserzählungen als rekonstruktive Gattung. In: Zeitschrift für Soziologie, 17:19-33. 
